International Journal of Biomedicine | June 2021 - Volume 11, Issue Suppl_1: Abstracts from the Third Russian International Conference "Cryo-electron microscopy 2021: achievements and prospects"

POSTER ABSTRACT PRESENTATIONS

SESSION TITLE: ADVANCES IN EM TECHNOLOGY AND PROCESSING

DOI: 10.21103/IJBM.11.Suppl_1.P1

\title{
Abstract P-1: Analysis of Tick-borne Encephalitis Virus Single-particle Imaging on X-ray Free-electron Laser
}

Grigoriy A. Armeev ${ }^{1}$, Alexey K. Shaytan ${ }^{1}$, Mikhail F. Vorovich ${ }^{2}$, Alexey M. Egorov $^{2}$, Aydar A. Ishmukhametov ${ }^{2}$, Mikhail P. Kirpichnikov ${ }^{1}$, Konstantin V. Shaitan ${ }^{1}$

${ }^{1}$ Department of Biology, Lomonosov Moscow State University, Moscow, Russia ${ }^{2}$ FSASI "Chumakov FSC R\&D IBP RAS”" (Institute of Poliomyelitis), Moscow, Russia

Background: Tick-borne encephalitis virus (TBEV) is a dangerous human pathogen which envelope structure is already known from cryoEM study. TBEV mature viral particle size $(\sim 50 \mathrm{~nm}$ in diameter) makes it suitable for singleparticle imaging (SPI) on X-ray free-electron laser (XFEL). XFEL SPI studies are at the early stages of development; thus, a well-described and conformationally homogeneous sample is required to develop approaches for experimental setup and data analysis. Here we present the image analysis results of data collected in October 2019 during the European XFEL experiment \#2316. Methods: The detector was placed at $1.62 \mathrm{~m}$ from the injector; photon energy was around $6 \mathrm{keV}$, pulse energy $4 \mathrm{~mJ}$, beam diameter $\sim 500 \mathrm{~nm}$. All runs were processed to detect hits with threshold filter (5th percentile of lit pixels) and further filtered to omit low-intensity images and images that lack detector modules. Filtered hits were background and geometry corrected with SPImage library and custom python scripts. Then hits were azimuthally integrated using PyFAI library. Scattering profiles were further clustered using the affinity propagation algorithm with cosine similarity metric in log space. Extracted classes were used to build averaged images. All hit profiles were fitted with model scattering to estimate the diameter of the particle. Simulated diffraction patterns were prepared using Condor from the cryoEM electron density map (EMDB ID 3752).

Results: During the analysis after the filtering, only 276 clean and bright hits were collected per 135 min of injection (from 27287 hits detected via lit pixels threshold). Thus the hit rate was around $\sim 2$ hits $/ \mathrm{min}$, which is expected to rise in the future. The majority of hits correspond to the $40-50 \mathrm{~nm}$ particles (Fig. 1a), 
which is expected for TBEV. However, the exact size may vary due to solvent evaporation, ion condensation, and possible variability in the sample.

Conclusion: The averaged images and their scattering profiles correlate with the simulated scattering patterns, though not ideally (Fig. 1 bc). Such discrepancy is expected due to the absence of electron density in the center of modeled viral structures.

a)

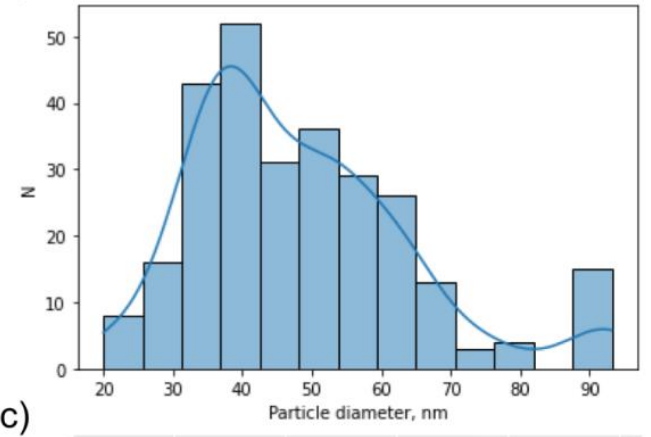

c)

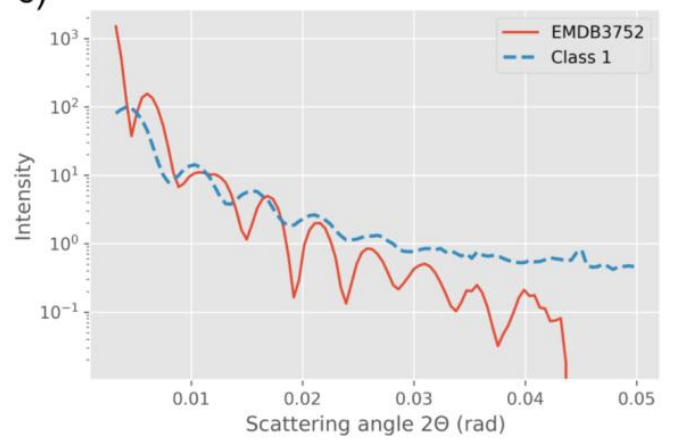

b)

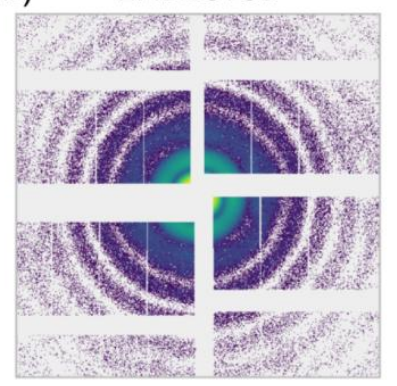

Class $4, \mathrm{~N}=16, \mathrm{D}=51.8 \mathrm{~nm}$

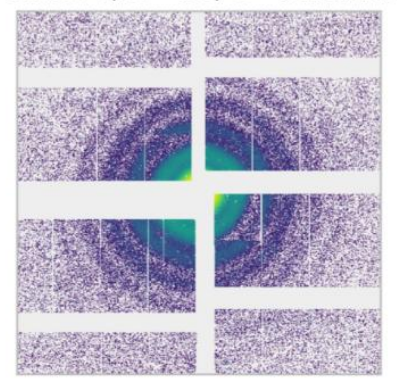

Class $1, \mathrm{~N}=21, \mathrm{D}=54.6 \mathrm{~nm}$

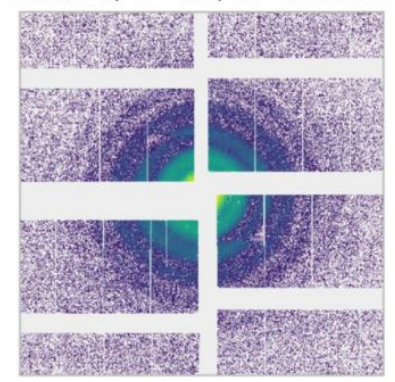

Class $11, \mathrm{~N}=11, \mathrm{D}=53.8 \mathrm{~nm}$

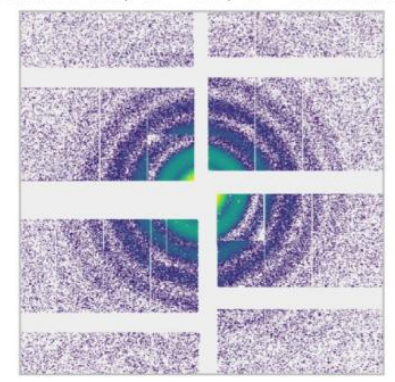

Fig. 1. a) Particles size distribution. b) Comparison of averaged images with modeled diffraction. c) Comparison of scattering profiles of averaged class 1 with diffraction modeled from cryoEM density map.

Key Words: XFEL・ single-particle imaging $\bullet$ viruses

This work was supported by the Russian Foundation for Basic Research (Grants No. 18-02-40010 (data analysis) and 18-02-40026 (sample preparation)).

*Corresponding author: Grigoriy Armeev.E-mail:armeev@intbio.org

International Journal of Biomedicine. 2021;11 Suppl 1: S11.

doi: 10.21103/IJBM.11.Suppl_1.P1

(C)2021 International Medical Research and Development Corporation 